\title{
ON THE LOCAL STRUCTURE OF THE TRAJECTORIES OF A QUADRATIC DIFFERENTIAL
}

\author{
JAMES A. JENKINS
}

1. Teichmüller was the first to bring out the important role played by quadratic differentials in extremal problems in the theory of func. tions. He indicated [3] the behavior in the small of certain important curves associated with them. It remained for Schaeffer and Spencer [2, Chap. III] to carry out this study in full detail. While they speak explicitly only of hyperelliptic differentials, the study of the local structure is the same in the general case. The object of the present paper is to show how, by a somewhat different approach to the problem, a considerable simplification is obtained of the technical details involved in this discussion.

In this local study we are concerned only with interior points of a Riemann surface. On an oriented Riemann surface $\Re$, open or closed, a quadratic differential is an entity which assigns to every local uniformizing parameter $z$ of $\Re$ a function $Q(z)$ meromorphic in the neighborhood associated with $z$ and satisfying the following condition. If $z^{*}$ is a second local uniformizing parameter of $\Re$ whose neighborhood on $\Re$ overlaps that of $z$ and $Q^{*}\left(z^{*}\right)$ is the corresponding function associated with $z^{*}$, then at common points of the neighborhoods of $z$ and $z^{*}$ we have

$$
Q^{*}\left(z^{*}\right)=Q(z)\left(\frac{d z}{d z^{*}}\right)^{2} .
$$

From this definition it is clear that we may speak of a quadratic differential having a zero or pole of a given order at a point of $\Re$ independently of the choice of local uniformizing parameter. A quadratic differential is to be denoted by the generic symbol $Q(z) d z^{2}$.

We see at once that the zeros and poles of a quadratic differential $Q(z) d z^{2}$ form a discrete set on $\Re$. The points of this set will be called singular points of $Q(z) d z^{2}$.

Of great importance are the curves on which $Q(z) d z^{2}>0$. Evidently these are independent of the choice of local uniformizing parameters. Maximal elements with this property will be called trajectories. Our present objective is to give the structure of the family of trajectories in the neighborhood of any point of $\Re$. This is relatively simple in case the point is nonsingular, a zero or a simple pole of

Received by the editors August 27, 1953. 
$Q(z) d z^{2}$. The desired result is then obtained by considering the (many-valued) function

$$
\zeta=\int(Q(z))^{1 / 2} d z
$$

in a neighborhood of the point. This is done in [2] and here we will only state the results.

A nonsingular point has a neighborhood which is swept out by open analytic arcs of trajectories. From this it follows that an individual trajectory is an open arc or Jordan curve on $\Re$.

A zero of $Q(z) d z^{2}$ of order $n$ is the limiting end point of $n+2$ trajectories which have limiting directions spaced at the angle $2 \pi /(n+2)$. A suitable neighborhood of the zero is divided by these into $n+2$ sectors swept out by open analytic arcs of trajectories.

A simple pole of $Q(z) d z^{2}$ is the limiting end point of a single trajectory which has a limiting direction at the pole.

2. The structure of the trajectories in the neighborhood of a pole of order $\geqq 2$ is more complicated and we now proceed to discuss this in detail. A trajectory may be regarded as an integral curve of the differential equation

$$
Q(z)\left(\frac{d z}{d \tau}\right)^{2}=1
$$

where $\tau$ can be interpreted as the real part of the function $\zeta$ of (1). This interpretation, coupled with the behavior of the trajectories near a nonsingular point, shows that, unless a trajectory has a limiting end point at a zero or simple pole, $\tau$ has on it the range $-\infty<\tau$ $<\infty$.

We take up first the case where the order of the pole exceeds 2 . We may suppose the pole to be given by $z=0$ for the local uniformizing parameter $z$.

Lemma 1. Let $Q(z)$ have a pole of order $\mu, \mu>2$, at $z=0$. There are $\mu-2$ limiting directions in which trajectories can approach the pole and these directions are equally spaced at angles of $2 \pi /(\mu-2)$. For $\alpha>0$, sufficiently small, every trajectory which meets $|z|<\alpha$ in each sense either tends to $z=0$ or leaves $|z|<\alpha$. If a trajectory lies entirely in $|z|<\alpha$, by adjoining to it the point $z=0$ we obtain a Jordan curve whose interior angle at $z=0$ is $2 \pi /(\mu-2)$. For $\alpha$ as above there exists a number $\rho(\alpha)$ such that if a trajectory meets $|z|=\rho, \rho \leqq \rho(\alpha)$, it tends in at least one sense to $z=0$ as limit. 
First suppose $\mu$ is even, $=2 m, m>1$. Then the differential equation (2) can be written as

$$
\frac{d z}{d \tau}=A z^{m}\left(1+b_{1} z+b_{2} z^{2}+\cdots\right)
$$

where we have made an arbitrary choice of square root (which does not affect the trajectories) and the series converges in some circle $|z|<R$ (in which $z$ acts as local uniformizing parameter). We subject $\alpha$ initially to the conditions that there is no singular point of $Q(z) d z^{2}$ on $|z| \leqq \alpha$ other than $z=0$ and that $\alpha<R$.

Now we substitute $z=(-A(m-1) Z)^{-1 /(m-1)}$ with the root chosen so that the differential equation becomes

$$
\frac{d Z}{d \tau}=1+c_{1} Z^{-1 /(m-1)}+c_{2} Z^{-2 /(m-1)}+\cdots .
$$

The above mapping carries $|z|<\alpha$ into an $(m-1)$-fold branched element exterior to a circle $|Z|=\beta$ and the series in $Z^{-1 /(m-1)}$ converges on the latter. Let now $\alpha$ be chosen small enough so that for $|Z| \geqq \beta$

$$
\left|c_{1} Z^{-1 /(m-1)}+c_{2} Z^{-2 /(m-1)}+\cdots\right|<1 / 2
$$

for each choice of the root. Set $Z=X+i Y$. Then on the image of a trajectory we have $d X / d \tau>1 / 2, d Y / d \tau<1 / 2$. Thus insofar as the image of a trajectory lies on the branched element exterior to $|Z|=\beta$, $X$ varies monotonically with $\tau$ and the curve has slope $<1$. The first statement implies that, in a given sense, the curve either tends to $Z=\infty$ or leaves $|Z|>\beta$. If the curve tends to $Z=\infty$ it is clear that on it $d Y / d \tau$ tends to zero and thus the curve tends to $Z=\infty$ in the direction of the positive or the negative real axis. Taken on the $(m-1)$ fold branched element these give $2 m-2$ limiting directions.

If the image of a trajectory meets $|Z|=2^{1 / 2} \beta$ at a point where $\Re Z \geqq 0$, then the fact that the curve has slope $<1$ shows that in the sense in which $X$ increases the curves cannot meet $|Z|=\beta$, thus must tend to $Z=\infty$. Similarly if the image of a trajectory meets $|Z|=2^{1 / 2} \beta$ at a point where $\Re Z \leqq 0$ the curve must tend to $Z=\infty$ in the sense in which $X$ decreases.

If the image of a trajectory lies entirely in $|Z|>\beta$ it tends to $Z=\infty$, in one sense in the direction of the positive real axis and in the other sense in the direction of the negative real axis, these lying in a single sheet of the $(m-1)$-fold branched element.

Translating these results back to the $z$-plane we obtain all the statements of Lemma 1 in the case where $\mu$ is even. As shown in [2] the case where $\mu$ is odd can be reduced trivially to the preceding. 
Lemma 2. Let $Q(z)$ have a pole of order 2 at $z=0$ and let $(Q(z))^{-1 / 2}$ have the expansion (for one choice of the root)

$$
(Q(z))^{-1 / 2}=(a+i b) z\left\{1+b_{1} z+b_{2} z^{2}+\cdots\right\}
$$

about $z=0$.

Case I, $a \neq 0, b \neq 0$ : For $\alpha>0$, sufficiently small, every trajectory which meets $|z|<\alpha$ tends in the one sense to $z=0$, and in the other sense leaves $|z|<\alpha$. Both modulus and argument of $z$ vary monotonically on the trajectory in $|z|<\alpha$. Each trajectory spirals about $z=0$, behaving asymptotically like a logarithmic spiral.

Case II, $a \neq 0, b=0$ : For $\alpha>0$, sufficiently small, every trajectory which meets $|z|<\alpha$ tends in the one sense to $z=0$ and in the other sense leaves $|z|<\alpha$. The modulus of $z$ varies monotonically on the trajectory in $|z|<\alpha$. Each trajectory has a limiting direction at $z=0$ depending on the trajectory.

Case III, $a=0, b \neq 0$ : Given $\epsilon>0$, there exists $\alpha(\epsilon)>0$ such that for $\alpha \leqq \alpha(\epsilon)$ a trajectory which meets $|z|=\alpha$ is a Jordan curve which lies in the ring $\alpha(1+\epsilon)^{-1}<|z|<\alpha(1+\epsilon)$.

Here the differential equation (2) takes the form

$$
\frac{d z}{d \tau}=(a+i b) z\left\{1+b_{1} z+b_{2} z^{2}+\cdots\right\}
$$

with the series converging in some circle $|z|<R$ (in which $z$ acts as local uniformizing parameter). We subject $\alpha$ initially to the conditions that there is no singular point of $Q(z) d z^{2}$ on $|z| \leqq \alpha$ other than $z=0$ and that $\alpha<R$.

Substituting $z=e^{Z}$, the differential equation becomes

$$
\frac{d Z}{d \tau}=a+i b+c_{1} e^{Z}+c_{2} e^{2 Z}+\cdots
$$

Under the above mapping the left-hand half-plane $\Re Z \leqq \log \alpha$ corresponds to $0<|z| \leqq \alpha$ covered by a logarithmic branch element. The series occurring here converges for $\Re Z \leqq \log \alpha$. We again set $Z=X$ $+i Y$.

Case I. Choosing $\alpha$ so small that for $\Re Z \leqq \log \alpha$

$$
\left|c_{1} e^{Z}+c_{2} e^{2 Z}+\cdots\right|<\min (|a| / 2,|b| / 2),
$$

we have $|d X / d \tau|>|a| / 2,|d Y / d \tau|>|b| / 2$ so that $X$ and $Y$ vary monotonically with $\tau$ on the portion of the image of a trajectory in $X<\log \alpha$. Further, on this curve $|d Y / d X|<3|b / a|$ so that the curve tends in one sense to $Z=\infty$ and in the other leaves $X<\log \alpha$. 
Setting $W=(a+i b)^{-1} Z, W=U+i V$ we have

$$
\frac{d V}{d \tau}=\Re\left\{b_{1} e^{Z}+b_{2} e^{2 Z}+\cdots\right\}
$$

Thus

$$
\left|\frac{d V}{d X}\right| \leqq M e^{X}
$$

for a constant $M>0$ in $X<\log \alpha$. Thus the variation of $V$ on the portion of the image of a trajectory to the left of the ordinate $X=t$ tends to zero as $t \rightarrow-\infty$. Hence the curve in question has as asymptote a line with slope $b / a$.

Translating these results back to the $z$-plane we obtain the statements of Lemma 2 in Case I.

Case II. The results in this case follow by an obvious modification of the methods used in Case I.

Case III. By the differential equation

$$
\frac{d Z}{d \tau}=i b+c_{1} e^{Z}+c_{2} e^{2 Z}+\cdots
$$

if we choose $\beta$ so that for $X \leqq \beta$

$$
\left|c_{1} e^{Z}+c_{2} e^{2 Z}+\cdots\right|<|b| / 2
$$

we see that on the portion of the image of a trajectory in $X<\beta, Y$ varies monotonically with $\tau$ and $|d X / d Y|<1$. Thus a portion of an image of a trajectory which meets $Y=t, 2 n \pi \leqq t \leqq 2(n+1) \pi$, to the left of $X=\beta-2 \pi$ meets $Y=2 n \pi$ and $Y=2(n+1) \pi$ in $X<\beta$. Now write the differential equation in the form

$$
i b\left(1+a_{1} e^{Z}+a_{2} e^{2 Z}+\cdots\right) \frac{d Z}{d \tau}=1
$$

and choose $\gamma$ so that

$$
\left|\Re\left\{\sum_{n=1}^{\infty} n^{-1} a_{n}\left(e^{n X_{2}}-e^{n X_{1}}\right)\right\}\right|<\left|X_{2}-X_{1}\right|
$$

for $X_{1} \neq X_{2}, X_{1}, X_{2}<\gamma$. (That this is possible is easily verified.) Suppose that an arc of the image of a trajectory joins the points $X_{1}$ $+2 m \pi i, X_{2}+2(m+1) \pi i$ where $X_{1}, X_{2}<\gamma$. Then integrating the differential equation along the arc and taking the imaginary part we find 


$$
\Re\left\{\sum_{n=1}^{\infty} n^{-1} a_{n}\left(e^{n X_{2}}-e^{n X_{1}}\right)\right\}+X_{2}-X_{1}=0
$$

which implies $X_{1}=X_{2}$. Hence if the image of a trajectory meets $X<\delta$, $\delta=\min (\beta, \gamma)-2 \pi$, the trajectory is a Jordan curve.

Now given $\eta>0$ let $\alpha(\eta)>0$ be such that $\log \alpha(\eta)<\delta$ and for $X<\log \alpha(\eta)+\eta$

$$
\left|c_{1} e^{z}+c_{2} e^{2 Z}+\cdots\right|<\frac{\eta|b|}{\eta+2 \pi} .
$$

Then on the image of a trajectory in $X<\log \alpha(\eta)+\eta$ we have $|d X / d Y|<\eta / 2 \pi$. If such a curve meets $X=\log \alpha, \alpha \leqq \alpha(\eta)$, the corresponding trajectory is a Jordan curve and the variation of $X$ on the image does not exceed $\eta$, hence it lies between $X=\log \alpha-\eta$ and $X=\log \alpha+\eta$.

Translating these results back to the $z$-plane we obtain the statements of Lemma 2 in Case III.

Our description of the local structure of the trajectories differs slightly from that of [2, Chap. III] but the statements there (Lemmas IX and X) could be proved just as easily. Not only does the present method supply a simpler approach (except perhaps in Case III of Lemma 2) but it is useful in investigating sharper properties of the structure of trajectories, cf. [1].

\section{BiBLIOGRAPHY}

1. James A. Jenkins, On a problem of Gronwall, to appear in Ann. of Math.

2. A. C. Schaeffer and D. C. Spencer, Coefficient regions for schlicht functions, Amer. Math. Soc. Colloquium Publications, vol. 35, 1950.

3. O. Teichmüller, Ungleichungen zwischen den Koeffizienten schlichter Funktionen, Preuss. Akad. Wiss. Sitzungsber. (1938) pp. 363-375.

JoHNS Hopkins University 